\title{
DUALITY IN PRODUCTS OF GROUPS WITH OPERATORS
}

\author{
BY \\ J. W. ELLIS( $(1)$
}

1. Introduction. The concept of a complementary group to a given group $X$, i.e., a group which may be considered as a certain collection of homomorphisms on $X$ to a (fixed) group $Z$, occurs in many places, as does that of dual groups, each of which is complementary to the other. Perhaps the most common examples of complementary groups are the character group of a topological group and the adjoint space of a linear topological space.

A natural question is: can the complementary group of a (weak or complete) cartesian product of groups be described easily in terms of the complements of the individual groups; in particular, is it some sort of product of these? This was answered affirmatively for certain groups and their character groups by Kaplan [4] and for adjoint spaces of locally-convex real linear Hausdorff spaces by Katětov [5].

This paper gives an affirmative answer in a situation general enough to include both of these results as well as some others. The investigation is done by using $S$-spaces and $S$-homomorphisms ( $\$ 2)$ where an $S$-space is an abelian group which has $S$ as a semigroup of operators. Groups are $J$-spaces, with $J$ the integers, and linear spaces over $\Phi$ are $\Phi$-spaces.

Our main theorem (4.4) states results about certain subspaces of a product which contain the weak product; its principal consequences state that, under certain conditions, the complement of a cartesian product of spaces, with the usual product topology, is the weak product of their complements; and the complement of a weak product of spaces, if its topology lies within a certain interval, is the cartesian product of the complements. Combining these in an obvious way provides duality theorems, including (8.5) Kaplan's result that the family of groups which are the character groups of their character groups is.closed under the formation of cartesian products, and (9.6) Katertov's theorem that the adjoint of a product is the weak product of the adjoints and dually.

2. Definitions and notation. The letter $S$ will denote a fixed, commutative semigroup, written multiplicatively. The symbols $C, R, J$ will represent either

Presented to the Society, November 28, 1952, under the title Complementary spaces of Cartesian products of groups and linear spaces; received by the editors February 4, 1955, and, in revised form, March 31, 1956.

(1) Atomic Energy Commission predoctoral Fellow, during a part of the preparation of this paper. Another part of this work was done under Contract N7-onr-434, Task Order III, Navy Department (Office of Naval Research). This is a portion of a dissertation presented to the Graduate School of Tulane University July 21, 1952, in partial fulfillment of the requirements for the degree of Doctor of Philosophy. 
the multiplicative semigroup or the additive group of the complexes, the reals and the integers, respectively; it will be clear from the context which is intended. We will designate by $X^{Y}$ the set of all functions on $Y$ to $X$; the circle group will be written as the quotient group $R / J$.

By an $S$-space we shall mean an abelian group $X$ (written additively and with identity $\theta$ ) upon which the members of $S$ act as homomorphisms; i.e., there is a function $s x$ on $S \times X$ to $X$ such that $s(x+y)=s x+s y$ and $(r s) x$ $=r(s x)$ for $r, s \in S$ and $x, y \in X$. An $S$-space will be called topologized if it has a topology in which left translations are continuous; topological if it is a topological group and multiplication by $s$ is continuous for each $s \in S$. $S$ subspaces, $S$-homomorphisms and $S$-isomorphisms are defined in the obvious way.

Any abelian group is a $J$-space; the $J$-subspaces are the subgroups and $J$-homomorphisms are the ordinary homomorphisms. A linear space over a field $\Phi$ is a $\Phi$-space; the $\Phi$-subspaces and $\Phi$-homomorphisms are the linear subspaces and the distributive functions. We also note that a commutative ring $X$ is an $X$-space under the usual product; the $X$-subspaces are the ideals and the $X$-homomorphisms are group-homomorphisms for which $f(x y)=x \cdot f(y)$ $=y \cdot f(x)$; if the ring has a unit, each $X$-homomorphism on the ring to itself is merely multiplication by a fixed element.

The cartesian product of an arbitrary collection of $S$-spaces $\left\{X_{a}: a \in A\right\}$ can be made into an $S$-space in the natural way, and the symbols $P\left\{X_{a}\right.$ : $a \in A\}$ or $P X_{a}$ will henceforth denote this product; the symbols $P^{w}\left\{X_{a}\right.$ : $a \in A\}$ or $P^{w} X_{a}$ will denote the $S$-subspace ("weak" product) consisting of those members of the product which are finitely nonzero. For each $a \in A$, we define $\pi_{a}: P X_{a} \rightarrow X_{a}$ and $i_{a}: X_{a} \rightarrow P X_{a}$ by $\pi_{a}(x)=x_{a}$ and $\left[i_{a}(p)\right]_{a}=p,\left[i_{a}(p)\right]_{b}$ $=\theta_{b}$ if $b \neq a$. These are both clearly $S$-homomorphisms, and $i_{a}\left(X_{a}\right) \subset P^{w} X_{a}$.

If $X$ and $Y$ are $S$-spaces, $H(X, Y)$ will denote the set of $S$-homomorphisms on $X$ to $Y$; if they have topologies, $C(X, Y)$ will denote the continuous members of $H(X, Y)$. Note that $H(X, Y)$ is an $S$-subspace of $Y^{\mathbf{X}}$; if $Y$ is topological, $C(X, Y)$ is an $S$-subspace of $H(X, Y)$.

If $X, Y$ and $Z$ are $S$-spaces and $Y$ and $Z$ are topologized, we say that $X$ is $a Z$-complement of $Y$ if there is an $S$-homomorphism $\mu$ on $X$ onto $C(Y, Z)$. Simple examples of complements are of course character groups and adjoint spaces. If $X$ is also topologized, we say that $X$ and $Y$ are $Z$-dual if they are simultaneously $Z$-complements of each other; that is, if there is a bi-Shomomorphism $\Omega: X \times Y \rightarrow Z$ such that $\Omega(x, Y)=C(X, Z)$ for each $x \in X$ and $\Omega(X, y)=C(Y, Z)$ for each $y \in Y$. A locally-compact abelian Hausdorff group and its character group are $R / J$-dual with $\Omega(\chi, y)=\chi(y)$; similarly, a real or complex linear topological space is $R$-dual or $C$-dual to its adjoint if the adjoint's topology is suitably selected. Finally, a discrete commutative ring $X$ with identity is $X$-dual to itself, with $\Omega\left(x, x^{\prime}\right)=x x^{\prime}$.

3. Additive ideals; the fundamental lemma. In what follows, $A$ will de- 
note some fixed set, and $\mathcal{F}$ will represent the family of finite subsets of $A$. By an additive ideal or U-ideal we will mean a hereditary family of subsets of $A$ which is closed under finite unions. For any $B C 2^{A}$, we define a complement $\mathbb{B}^{\prime}=\{E: E \subset A$ and $E \cap B \in \mathcal{F}$ for every $B \in \mathbb{B}\}$. Also, let $\mathbb{B}^{\prime \prime}=\left(\mathbb{B}^{\prime}\right)^{\prime}$. Clearly $\mathbb{B}^{\prime}$ is always a $U$-ideal and $F \subset B^{\prime}$; also, $B \subset B^{\prime \prime}$ but equality need not hold even if $B$ is itself a $U$-ideal.

For each $a \in A$, let $X_{a}$ be an $S$-space; if $x \in P X_{a}$, let $A(x)=\{a: a \in A$, $\left.\pi_{a}(x) \neq \theta_{a}\right\}$; then, for any $B \subset 2^{A}$, let $L(B)=\left\{x: x \in P X_{a}\right.$ and $\left.A(x) \in B\right\}$. If $B$ is a $U$-ideal, $L(B)$ is an $S$-subspace of $P X_{a}$.

Several examples of $U$-ideals and their complements may be mentioned here: (i) If $B=2^{A}$, then $B^{\prime}=\mathcal{F}$ and $B^{\prime \prime}=B$; in this case, $L(B)=P X_{a}$ and $L\left(\mathbb{B}^{\prime}\right)=P^{w} X_{a}$; (ii) If $A$ is a locally-compact Hausdorff space, and $B$ the family of precompact subsets, then $B^{\prime}$. consists of all closed discrete subsets; if $A$ is also $\sigma$-compact, $\mathbb{B}^{\prime \prime}=\mathbb{B}$; here $L(B)$ consists of the members of the product which have "compact supports"; (iii) If $I$ denotes the positive integers, and $A=I \times I$, take $B=\{B: B \subset A, B \subset F \times I$ for some finite $F\}$; then $B$ is a $U$-ideal and $B^{\prime}=\left\{M\right.$ : for each $n \in I$, there is $F_{n}$ finite so that $M \subset \cup\{n\}$ $\left.X F_{n}: n \in I\right\}$; also $\mathbb{B}^{\prime \prime}=\mathbb{B}$ (this example is essentially due to Köthe [6, pp. 34-35]).

From now on, let $Z$ be a fixed $S$-space, and $B$ a fixed $U$-ideal of subsets of $A$. For each $a \in A$, let $X_{a}, Y_{a}$ be $S$-spaces and $\mu^{a}$ a homomorphism on $X_{a}$ into $H\left(Y_{a}, Z\right)$. Set $X=L\left(\cap^{\prime}, P X_{a}\right)$ and $Y=L\left(囚, P Y_{a}\right)$ and then define $\mu: X \rightarrow Z^{Y}$ by

$$
[\mu(x)](y)=\sum\left\{\left[\mu^{a}\left(x_{a}\right)\right]\left(y_{a}\right): a \in A\right\} .
$$

This function is well-defined, is an $S$-homomorphism into $H(Y, Z)$, and is 1-1 if and only if each $\mu^{a}$ is.

3.1. Fundamental Lemma. If $f$ is a group-homomorphism on $Y$ to $Z$, if the composition $f \circ i_{a} \in \mu^{a}\left(X_{a}\right)$ for each $a \in A$, and if there is $B^{\prime} \in B^{\prime}$ such that $f(y)=\theta$ whenever $A(y) \cap B^{\prime}=\Lambda$, then $f=\mu(x)$ for some $x \in X$.

Proof. For each $a \in A$, choose $q(a) \in X_{a}$ such that $\mu^{a}[q(a)]=f \circ i_{a}$. Define $\bar{x} \in P X_{a}$ by $\bar{x}_{a}=q(a)$ for $a \in B^{\prime}, x_{a}=\theta_{a}$ for $a \notin B^{\prime}$; then $\bar{x} \in X$ and we will show that $\mu(\bar{x})=f$. For any $y \in Y, A(y) \in B$; thus $A(y) \cap B^{\prime}$ is a finite set $G$. If $y^{\prime}=y-\sum\left\{i_{a}\left(y_{a}\right): a \in G\right\}$, then $y^{\prime} \in Y$ and $A\left(y^{\prime}\right) \cap B^{\prime}=\Lambda$; hence $f\left(y^{\prime}\right)=\theta$, or

$$
f(y)=\sum\left\{f\left[i_{a}\left(y_{a}\right)\right]: a \in G\right\}=\sum\left\{\left[\mu^{a}\left(\bar{x}_{a}\right)\right]\left(y_{a}\right): a \in A(y)\right\}=[\mu(\bar{x})](y) .
$$

4. Topologies for $Y$; the main theorem. In this and the subsequent sections we assume the same situation described in the preceding section; in addition, we assume that each $Y_{a}$ is topologized, that $Z$ is topological and that each $\mu^{a}$ is onto $C\left(Y_{a}, Z\right)$. For $M \subset X, N \subset Z$, define $G(M, N)=\{y$ : $[\mu(x)](y) \in N$ for $x \in M\}$.

Next we consider a collection of topologies any of which may be assigned 
to $Y$; the symbols introduced here will be used throughout the remainder of the paper. If $\mathcal{G}$ is any family of subsets of $Y$ each containing $\theta$, let $\mathcal{G}_{F}$ be the family of finite intersections of members of $\mathrm{G}$. We will define $T(\mathcal{G})$ as the topology for $Y$ in which a set $U$ is open if and only if for each $x \in U$ there is $G \in \mathcal{G}_{F}$ such that $x+G \subset U$. Then $(Y, T(\mathcal{G}))$ is a topologized $S$-space and, although the members of $G$ need not be nuclei, continuity of $S$-homomorphisms on or into $(Y, T(\mathcal{G}))$ may be verified by using $\mathcal{G}_{F}$ instead of the family of nuclei. We will use the following families:

$W=\{G(\{x\}, V): x \in X, V$ a nucleus in $Z\}$; $M\}$;

$\mathbb{P}=\left\{M\right.$ : for some $B^{\prime} \in \mathbb{B}^{\prime}$, any $y \in Y$ such that $A(y) \cap B^{\prime}=\Lambda$ belongs to

$\mathscr{T}=\left\{M:\right.$ for each $a \in A$, there is a nucleus $V_{a}$ in $Y_{a}$ such that $\left.i_{a}\left(V_{a}\right) \subset M\right\} ;$

$R=\left\{P V_{a} \cap Y\right.$ : each $V_{a}$ a nucleus in $\left.Y_{a}\right\}$;

$Q=\left\{P V_{a} \cap Y\right.$ : each $V_{a}$ a nucleus in $Y_{a}$ and all but a finite number equal to $\left.Y_{a}\right\}$.

If, in addition, $X$ has a topology, we define

$\mathfrak{e}=\{G(K, V): K$ compact in $X, V$ a nucleus in $Z\}$.

The topology $T(W)$ is usually called the weak topology, $T(\mathcal{C})$ the compactopen topology; $T(Q)$ is the (relativized) product topology; $T(R)$ may be called, as is done by Kaplan [4], the rectangular topology for $Y$. The reader may

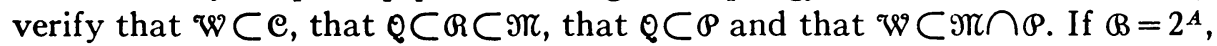
then $T(W) \subset T(Q)$; that is, every $T(W)$-open set is $T(Q)$-open.

We say that a topologized $S$-space $L$ does not have arbitrarily small $S$-subspaces if there is a nucleus in $L$ which contains no $S$-subspace other than $\theta$. We are now ready for the main theorem, whose proof is given in three lemmas; the first two proofs are left to the reader.

4.1. Lemma. If $\Im \supset T(W)$, then $\mu(X) \subset C[(Y, \Im), Z]$.

4.2. LEmma. If $\Im \subset T(\mathfrak{T})$, then $C[(Y, \Im), Z] \circ i_{a} \subset C\left(Y_{a}, Z\right)$ for each $a \in A$.

4.3. LEMMA. If $Z$ does not have arbitrarily small $S$-subspaces, and $\Im \subset T(\mathcal{P})$, then for any $f \in C[(Y, \Im), Z]$ there is $B^{\prime} \in B^{\prime}$ such that $f(y)=\theta$ whenever $A(y)$ $\cap B^{\prime}=\Lambda$.

Proof. Let $U$ be a nucleus in $Z$ containing no $S$-subspace other than $\theta$. If $f \in C[(Y, \Im), Z] \subset C[(Y, T(\mathcal{P})), Z]$, there is $B^{\prime} \in B^{\prime}$ such that $f(y) \in U$ whenever $A(y) \cap B^{\prime}=\Lambda$. The collection of all such $y$ is an $S$-subspace of $Y$; its image under $f$ is an $S$-subspace contained in $U$, which must then be $\theta$, proving the lemma.

4.4. THEOREM. If each $X_{a}$ is a Z-complement of $Y_{a}$ and $\mu^{a}$ is the corresponding $S$-homomorphism; if $Z$ does not have arbitrarily small $S$-subspaces; and if the topology $\mathfrak{J}$ for $Y$ is such that $T(\mathcal{W}) \subset J \subset T(\mathfrak{T}) \cap T(\beta)$, then $X$ is a $Z$-complement of $(Y, \mathfrak{J})$, under the $S$-homomorphism $\mu: X \rightarrow C[(Y, \Im), Z] d e$ fined in (1). 
Proof. Immediate from the three preceding lemmas and the Fundamental Lemma (3.1).

We remark that, since $W \subset \mathfrak{M} \cap \odot$, there are always such topologies. Two applications of this theorem will, of course, give a theorem on dual spaces.

4.5. THEOREM. If $B^{\prime \prime}=\bigotimes$ and, for each $a, X_{a}$ and $Y_{a}$ are $Z$-dual and $\Omega^{a}$ is the corresponding bihomomorphism; if $Z$ does not have arbitrarily small $S$ subspaces; and if $\mathfrak{J}, \mathcal{U}$ are topologies for $Y, X$ such that $T(W) \subset J \subset T(\mathfrak{T}) \cap T(\mathcal{P})$ and analogous relations hold for $\mathcal{U}$, then $(X, \mathcal{u})$ and $(Y, \mathfrak{J})$ are $Z$-dual, with a bihomomorphism on $X \times Y$ to $Z$ defined by

$$
\Omega(x, y)=\sum\left\{\Omega^{a}\left(x_{a}, y_{a}\right): a \in A\right\} .
$$

5. The full product. We now focus our attention on the two $U$-ideals which are of most frequent use: $\mathcal{F}$ and $2^{A}$.

If $B=2^{A}$, then $\mathbb{B}^{\prime}=\mathcal{F}, Y=P Y_{a}$ and $X=P^{w} X_{a}$, and it follows from earlier remarks that $T(Q)$, the usual product topology for $Y$, lies within the interval prescribed in Theorem 4.4; hence we can state the following result.

5.1. ThEOREM. If each $X_{a}$ is a Z-complement of $Y_{a}$, with corresponding homomorphism $\mu^{a}$, and if $Z$ does not have arbitrarily small $S$-subspaces, then $P^{w} X_{a}$ is a Z-complement of $P Y_{a}$ in the product topology, under the S-homomorphism $\mu$ on $P^{w} X_{a}$ to $C\left(P Y_{a}, Z\right)$ defined in (1).

6. The weak product. If $B=\mathcal{F}$, then $B^{\prime}=2^{A}, Y=P^{w} Y_{a}, X=P X_{a}$ and $T(\mathcal{P})$ is the discrete topology for $Y$. As before, we have $T(Q) \subset T(R) \subset T(\mathfrak{T})$, and $T(\mathscr{W}) \subset T(\mathcal{C}) \cap T(\mathfrak{T})$ when $T(\mathcal{C})$ is defined. The two succeeding lemmas concern other possible relationships between topologies.

6.1. LemmA. If $X$ and each $X_{a}$ are topologized in such a way that each projection $\pi_{a}$ is continuous, and if the topology of each $Y_{a}$ is finer than the compactopen topology (which it has as a subset of $Z^{X_{a}}$ ), then $\mathrm{C} \subset \mathrm{T}$.

Proof. Take $G=G(K, V) \in \mathrm{e}$. For each $a \in A$, let $K_{a}=\pi_{a}(K)$; then $K_{a}$ is compact in $X_{a}$ and $U_{a}=G\left(K_{a}, V\right)$ is a nucleus in $Y_{a}$. Since $[\mu(k)]\left(i_{a}(p)\right)$ $=\left[\mu^{a}\left(\pi_{a}(k)\right)\right](p) \in V$ for $k \in K$ and $p \in U_{a}$, we have $i_{a}\left(U_{a}\right) \subset G$ for each $a \in A$, proving the lemma.

6.2. Definition. The topological $S$-space $Z$ has property $G(X)$ if it has a nucleus $B$ and a nuclear base $V$ such that, for any $V \in V$, there is a positive integer $n$ such that $G(n K, B) \subset G(K, V)$ whenever $K$ is compact in $X$ and $\theta \in K$.

Remark. The spaces $R$ and $C$ have property $G(X)$ for any $R$-space (resp., $C$-space) $X$; take $B=\{x:|x|<1\}$ and $V$ to be the family of all convex nuclei. The circle group $R / J$ has property $G(X)$ for any topological group $X$; take $B=\{r:|r|<1 / 8\}$ and let $U$ be the family of all nuclei of the form $\left\{r:|r|<r_{0}\right\}$ with $r_{0}<1 / 4$. This last result may be easily verified by the argument used by Kaplan [4, Lemma 2.8]. 
6.3. LEMMA. If each $X_{a}$ is topologized and the topology of $Y_{a}$ is coarser than the compact-open topology (as a subset of $Z^{X_{a}}$ ); if $X$ has the product topology; and if $Z$ has property $G\left(X_{a}\right)$ for each $a \in A$, then $T(R) \subset T(\mathcal{C})$.

Proof. Take $R \in R$; it will suffice to show that there is $G \in \mathbb{C}$ such that $G \subset R$. Write $R=P U_{a} \cap Y$; for each $a \in A$, there is $K_{a}$ compact in $X_{a}$ and a nucleus $V_{a}$ in $Z$ such that $G\left(K_{a}, V_{a}\right) \subset U_{a}$; we may of course assume that $\theta_{a} \in K_{a}$ and choose $V_{a}$ in the special nuclear base given by property $G\left(X_{a}\right)$. Then there is a positive integer $n_{a}$ such that $G\left(n_{a} K_{a}, B\right) \subset G\left(K_{a}, V_{a}\right) \subset U_{a}$; let $K=P\left(n_{a} K_{a}\right)$, a compact set in $X$. To show that $G(K, B) \subset R$, take $y \in G(K, B)$; for each $a \in A$ and $p \in K_{a}$, we have $i_{a}\left(n_{a} p\right) \in K$, so that

$$
\left[\mu^{a}\left(n_{a} p\right)\right]\left(y_{a}\right)=\left[\mu\left(i_{a}\left(n_{a} p\right)\right)\right](y) \in B ;
$$

this shows that $y_{a} \in G\left(n_{a} K_{a}, B\right) \subset U_{a}$. Thus $y \in P U_{a} \cap Y=R$, and the proof is complete.

These two lemmas imply the following.

6.4. Lemma. If each $X_{a}$ is topologized, each $Y_{a}$ has the compact-open topology, $X$ has the product topology, and $Z$ has property $G\left(X_{a}\right)$ for each $a \in A$, then $T(Q) \subset T(R) \subset T(\mathcal{e}) \subset T(\mathfrak{N}) \subset T(\mathcal{P})$.

Since $T(\mathcal{P})$ is the discrete topology, Theorem 4.4 gives us the following result.

6.5. THEOREM. If each $X_{a}$ is a $Z$-complement of $Y_{a}$ with corresponding $S$ homomorphism $\mu^{a}$ and $\mathfrak{J}$ is a topology for $P^{w} Y_{a}$ such that $T(W) \subset J \subset T(\mathfrak{T})$, then $P X_{a}$ is a Z-complement of $\left(P^{w} Y_{a}, J\right)$ under the S-homomorphism $\mu: P X_{a}$ $\rightarrow C\left(P^{w} Y_{a}, Z\right)$ defined by (1).

We take time out for a result which is needed later ( $\$ 9)$.

6.6. Lemma. For any $x \in X$ and any nucleus $V$ in $Z$ there is $M \in \mathscr{N}$ such that $[\mu(x)](M) \subset V$.

Proof. For each $a \in A$, let $U_{a}=G\left(\left\{x_{a}\right\}, V\right)$, a nucleus in $Y_{a}$; let $M=\bigcup\left\{i_{a}\left(U_{a}\right): a \in A\right\}$. Then for $y=i_{a}(p) \in M$, we have $[\mu(x)](y)=\left[\mu^{a}\left(x_{a}\right)\right](p)$ $\in V$.

6.7. Lemma. Let $D, D^{\prime}$ be functions on $2^{Y}$ to $2^{Y}$ and $2^{Z}$ to $2^{Z}$, respectively, such that $D^{2}=D, D(M) \supset M$ for all $M \in \mathscr{M}$ and $h[D(E)] \subset D^{\prime}[h(E)]$ for any $h \in H(Y, Z)$ and $E \subset Y$. Suppose further that among the sets $E^{\prime} \subset Z$ such that $D^{\prime}\left(E^{\prime}\right)=E^{\prime}$ there is a nuclear base. Let $\mathfrak{N}=\{N: N \subset Y, D(N)=N\}$; then $T(W) \subset T(\mathfrak{T} \cap \Re) \subset T(\mathfrak{T})$.

Proof. The second inclusion is obvious. To prove the first, take $G(\{x\}, V)$ $\in W$. By hypothesis, there is a nucleus $U$ in $Z$ such that $U=D^{\prime}(U) \subset V$. By Lemma 6.6, there is $M \in \mathscr{M}$ such that $[\mu(x)](M) \subset U$. Let $N=D(M)$; then $N \in \mathfrak{T} \cap \Re$ and $[\mu(x)](N)=[\mu(x)](D(M)) \subset D^{\prime}([\mu(x)](M)) \subset D^{\prime}(U) \subset V$. 
The preceding lemmas show that $T(\mathbb{C})$ and $T(\Re \cap \Re)$ are among the topologies which may be used for $J$ in Theorem 6.5.

7. The compact-open topology for the weak product. We continue to assume that $B=\mathcal{F}$, and thus $Y=P^{w} Y_{a}$.

7.1. Lemma. Suppose each $Y_{a}$ is Hausdorff, and $J$ is a topology for $Y$ such that $J \supset T(R)$. Then for any set $K$ compact in $(Y, \Im)$ the set $P(K)=\{a: a \in A$, $\left.\pi_{a}(K) \neq \theta_{a}\right\}$ is finite.

Proof. (Following Kaplan [4, Theorem 2]). Suppose $P(K)$ is infinite; we may choose, for each $a \in P(K)$, a point $m(a) \in K$ such that $\pi_{a}(m(a)) \neq \theta_{a}$. Let $K^{\prime}=\{m(a): a \in P(K)\} \subset K$; then $K^{\prime}$ is infinite, since $A(k)$ is finite for $k \in Y$, whereas $\cup\left\{A(k): k \in K^{\prime}\right\}=P(K)$ is infinite. We will show that no point of $K$ can be an accumulation point of $K^{\prime}$; this will contradict the compactness of $K$, proving the lemma. Take $k \in K$. For each $a \in A$, choose a nucleus $U_{a}$ in $Y_{a}$ as follows: if $a \notin P(K)$ and $a \in A(k)$ select $U_{a}$ so that $\pi_{a}(m(a)) \notin U_{a}$; otherwise let $U_{a}=Y_{a}$. Then $R=P U_{a} \cap Y$ is a $T(R)$-nucleus and thus a J-nucleus. Clearly $k \in R$, but $m(a) \in R$ only if $a \in A(k)$, a finite set; hence $R \cap K^{\prime}$ is finite and $k$ is not an accumulation point of $K^{\prime}$.

7.2. Theorem. Let each $Y_{a}$ be Hausdorff and $X_{a}$ be a Z-complement of $Y_{a}$ topologized with the corresponding compact-open topology (each $\mu^{a}$ is then an $S$ isomorphism). Let $u$ be the product topology for $X=P X_{a}$ and $\Im$ a topology for $Y=P^{w} Y_{a}$ such that $T(\mathcal{W}) \cup T(R) \subset J \subset T(\mathfrak{N})$. Then $\mu$, as defined in (1), is an $S$-isomorphism on $X$ onto $C[(Y, J), Z]$ and is a homeomorphism if the latter has the compact-open topology.

Proof. It is clear from previous theorems that $\mu$ is an $S$-isomorphism onto; we will prove that it is bicontinuous.

Let $P U_{a}$ be a $u$-nucleus in $X$. For each $a \in A, U_{a} \supset G\left(K_{a}, V_{a}\right)$ for some compact $K_{a}$ in $Y_{a}$ and nucleus $V_{a}$ in $Z$; without loss of generality, we may assume that $\theta_{a} \in K_{a}$ and that $K_{a}=\theta_{a}$ except for $a$ in a finite set $F \subset A$. Let $K=\bigcup\left\{i_{a}\left(K_{a}\right): a \in A\right\}=\bigcup\left\{i_{a}\left(K_{a}\right): a \in F\right\}$; since $\Im \subset T(\mathfrak{T}), K$ is compact in ( $Y$, J). Let $V=\bigcap\left\{V_{a}: a \in F\right\}$, which is a nucleus in $Z$; then $G(K, V)$ is a compactopen nucleus in $C[(Y, \Im), Z]$ and $\mu^{-1} G(K, V) \subset P U_{a}$, for if $h \in G(K, V)$ and $p \in K_{a}$ for some $a$, we have

$$
\left[\mu^{a} \pi_{a} \mu^{-1}(h)\right](p)=\left[\mu \mu^{-1}(h)\right]\left(i_{a}(p)\right)=h\left(i_{a}(p)\right) \in V_{a}
$$

so that $\pi_{a} \mu^{-1}(h) \in G\left(K_{a}, V_{a}\right) \subset U_{a}$, or $\mu^{-1}(h) \in P U_{a}$. This proves that $\mu^{-1}$ is continuous.

Now let $G(K, V)$ be any compact-open nucleus in $C[(Y, \mathfrak{J}), Z]$; once again we may assume $\theta \in K$. By Lemma 7.1, $\pi_{a}(K)=\theta_{a}$ except for $a$ in a finite set $F \subset A$. Since $J$ is finer than the product topology $T(Q)$, each set $\pi_{a}(K)$ is compact in $Y_{a}$. If $F$ has $m$ points, choose a nucleus $U$ in $Z$ such that $m U \subset V$. Set $U_{a}=\left\{p: p \in X_{a}\right.$ and $\left.\left[\mu^{a}(p)\right]\left(\pi_{a}(K)\right) \subset U\right\}$; this is a nucleus, and is $X_{a}$ for 
$a \notin F$. Thus $P U_{a}$ is a $u$-nucleus in $X$; we will show that $\mu\left(P U_{a}\right) \subset G(K, V)$. If $x \in P U_{a}$ and $k \in K$,

$$
\begin{aligned}
{[\mu(x)](k) } & =\sum\left\{\left[\mu^{a}\left(x_{a}\right)\right]\left(k_{a}\right): a \in A\right\} \\
& =\sum\left\{\left[\mu^{a}\left(x_{a}\right)\right]\left(\pi_{a}(k)\right): a \in F\right\} \in m U \subset V .
\end{aligned}
$$

This concludes the proof.

As noted earlier, there are conditions under which $T(R) \subset T(\mathcal{C}) \subset T(\mathfrak{T})$; hence the following corollary.

7.3. Corollary. Suppose, for each $a \in A$, that $X_{a}$ is a $Z$-complement of $Y_{a}$, that $X_{a}$ and $Y_{a}$ have the compact-open topology each relative to the other, that each $Y_{a}$ is Hausdorff, and that $Z$ has property $G\left(X_{a}\right)$. Let $U$ be the product topology for $P X_{a}$ and $J$ the compact-open topology for $C\left[\left(P^{w} Y_{a}, T(\mathcal{e})\right), Z\right]$. Then the function $\mu:\left(P X_{a}, \mathfrak{U}\right) \rightarrow\left\{C\left[\left(P^{w} Y_{a}, T(\mathcal{C})\right), Z\right], \mathfrak{J}\right\}$, as defined by (1) is an S-isomorphism and homeomorphism onto.

From Theorem 4.5 , with $B=\mathcal{F}$, we obtain this duality theorem:

7.4. Theorem. Suppose $Z$ does not have arbitrarily small $S$-subspaces and, for each $a \in A, X_{a}$ and $Y_{a}$ are $Z$-dual $S$-spaces connected by the bihomomorphism $\Omega^{a}$. If $\mathcal{U}$ is the product topology for $P X_{a}$ and $\Im$ is a topology for $P^{w} Y_{a}$ such that $T(W) \subset \Im \subset T(\Re)$, then $\left(P X_{a}, \mathcal{U}\right)$ and $\left(P^{w} Y_{a}, J\right)$ are $Z$-dual, with corresponding bihomomorphism $\Omega$ defined as in (2).

8. Applications to topological groups. In this section, we indicate how our previous results may be applied to topological groups, and we list a few of the theorems thus obtained. The semi-group $S$ is always taken to be the integers $J$; all spaces involved are groups considered as $J$-spaces. The space $Z$ will always be $R / J$, which is a topological $J$-space, does not have arbitrarily small subgroups and, as remarked earlier, has property $G(X)$ for any topological group $X$.

If $G$ is any topological group (TG), the group $C(G, R / J)$ will be denoted, as usual, by $G^{*}$. The identity function on $G^{*}$ is a $J$-isomorphism which shows that $G^{*}$ is an $R / J$-complement of $G$. In this setting, the principal results of $\S \S 5-6$ give us the theorems which follow.

8.1. Theorem. Let $G_{a}$ be a $T G$ for each $a \in A$. If $u$ is the product topology for $P G_{a}$, then $P^{w} G_{a}^{*} \cong\left(P G_{a}, \mathcal{U}\right)^{*}$; if $\mathcal{J}$ is any topology for $P^{w} G_{a}$ such that $\dot{T}(\mathfrak{W}) \subset \mathfrak{J} \subset T(\mathfrak{T T})$, then $P G_{a}^{*} \cong\left(P^{w} G_{a}, \mathfrak{J}\right)^{*}$. The isomorphism $\mu$, in either case, is given by $[\mu(x)](y)=\sum\left\{x_{a}\left(y_{a}\right): a \in A\right\}$.

8.2. TheOREM. For each $a \in A$, let $G_{a}$ and $H_{a}$ be $T G$ s which are character groups of each other. If $u$ is the product topology for $P G_{a}$ and $\mathfrak{J}$ a topology for $P^{w} H_{a}$ such that $T\left({ }^{w}\right) \subset \mathfrak{J} \subset T(\mathfrak{T})$, then each of the groups $\left(P G_{a}, \mathcal{u}\right)$ and $\left(P^{w} H_{a}\right.$, $J)$ is algebraically isomorphic to the character group of the other.

8.3. Definition. Two TGs will be called Pontrjagin-dual if each is iso- 
morphic and homeomorphic to the character group of the other (with the usual compact-open topology in the character groups). Such groups are of course $R / J$-dual in our sense.

Corollary 7.3 now yields Theorem 8.4 and its corollary.

8.4. Theorem. For each $a \in A$, let $G_{a}, H_{a}$ be a pair of Pontrjagin-dual Hausdorff groups with bihomomorphism $\Omega^{a}$. Let $T(\mathcal{C})$, as before, designate the compact-open topology for $P^{w} H_{a}$ (as functions on $P G_{a}$ ). Then, if $u$ is the product topology for $P G_{a}$, the groups $\left(P G_{a}, \mathfrak{U}\right)$ and $\left(P^{w} H_{a}, T(\mathcal{C})\right)$ are Pontrjagin-dual, connected by the bihomomorphism $\Omega(g, h)=\sum\left\{\Omega^{a}\left(g_{a}, h_{a}\right): a \in A\right\}$.

8.5. Corollary. The family of Hausdorff groups which are (algebraically and topologically) the character groups of their character groups is closed under the operation of taking arbitrary cartesian products.

This corollary is essentially the main result of Kaplan's first paper [4] on extensions of the Pontrjagin duality, as pointed out in his introduction. Kaplan, in addition, describes a base, in $P^{w} G_{a}^{*}$, for the topology $T(\mathfrak{e})$, which he calls the "asterisk topology."

9. Applications to linear topological spaces. In this section, we will indicate how the results of $\S \S 5-6$ may be applied to real or complex linear topological spaces (LTS), and will list several of the principal results. The semigroup $S$ will always be either the real numbers, $R$, or the complex numbers, $C$; all spaces will be LTS, considered as topological $R$-spaces or $C$-spaces, as the case may be.

There are many LTS which do not have arbitrarily small linear subspaces; among these are the Hausdorff LTS which are locally-bounded in the sense of Hyers [3], a class which includes the normed linear spaces. From Theorems 5.1 and 6.5 we have the following.

9.1. THEOREM. Let $Z$ be an LTS without arbitrarily small linear subspaces; for each $a \in A$, let $Y_{a}$ be an LTS and $X_{a}=C\left(Y_{a}, Z\right)$. If $\Xi$ is the product topology for $P Y_{a}$, then $P^{w} X_{a}$ is isomorphic to $C\left[\left(P Y_{a}, \Im\right), Z\right]$, with isomorphism $\mu$ defined by $[\mu(x)](y)=\sum\left\{x_{a}\left(y_{a}\right): a \in A\right\}$.

9.2. TheOREM. Let $Z$ be any LTS; for each $a \in A$, let $Y_{a}$ be an LTS and $X_{a}=C\left(Y_{a}, Z\right)$. If $I$ is a topology for $P^{w} Y_{a}$ such that $T(W) \subset J \subset T(\mathfrak{T})$, then $P X_{a}$ is isomorphic to $C\left[\left(P^{w} Y_{a}, \mathfrak{J}\right), Z\right]$, under the isomorphism $\mu$ defined by $[\mu(x)](y)=\sum\left\{x_{a}\left(y_{a}\right): a \in A\right\}$.

9.3. Definition. Let $Z$ and each $Y_{a}$ be a real LTS; in this case we define two additional families of subsets of $P^{w} Y_{a}$ :

$\mathcal{E}=\{E: E$ convex, $E \in \mathscr{T}\}$;

$\mathcal{S}=\{G: G \in \mathcal{E}, G$ radially-open at $\theta\}$.

The topology $T(\mathcal{G})$ is used by Katêtov [5]. We have from 6.7 the theorem which follows. 
9.4. Theorem. Let $Z$ be a locally-convex real LTS. For each $a \in A$, let $Y_{a}$ be a real LTS. Then (i) $\mathcal{E}=\mathcal{G}$ and (ii) if $X_{a}=C\left(Y_{a}, Z\right)$, then $P X_{a}$ is isomorphic to $C\left[\left(P^{w} Y_{a}, T(\mathcal{E})\right), Z\right]=C\left[\left(P^{w} Y_{a}, T(\mathcal{G})\right), Z\right]$.

Proof. Take $E \in \mathcal{E}$; we will show that $E$ is radially-open at $\theta$. For each $a \in A$, there is a nucleus $U_{a}$ in $Y_{a}$ such that $i_{a}\left(U_{a}\right) \subset E$; since $Y_{a}$ is an LTS, we may choose $U_{a}$ to be radially-open at $\theta_{a}$. Take $y \in P^{w} Y_{a}$; then $y=\sum\left\{i_{a}\left(y_{a}\right): a \in F\right\}$, where $F=A(y)$ is finite, say with $m$ elements. For each $a \in F$, there is some $\lambda_{a}>0$ such that, if $|\beta|<\lambda_{a}$, then $\beta y_{a} \in U_{a}$. Let $\lambda=(1 / m)$ $\cdot\left(\min \left\{\lambda_{a}: a \in F\right\}\right)$. Then $\lambda>0$ and if $|\beta|<\lambda$, we have $m \beta y_{a} \in U_{a}$ for each $a \in F$; therefore,

$$
\beta y=\beta\left(\sum\left\{i_{a}\left(y_{a}\right): a \in F\right\}\right)=(1 / m)\left(\sum\left\{i_{a}\left(m \beta y_{a}\right): a \in F\right\}\right)
$$

and this belongs to the convex set $E$, proving (i).

To prove (ii), let $D(E), D^{\prime}\left(E^{\prime}\right)$ represent the convex covers of $E \subset Y$ and $E^{\prime} \subset Z$, respectively. Since $D, D^{\prime}$ satisfy the hypotheses of Lemma 6.7, and $\mathfrak{N} \cap \Re=\varepsilon=\mathcal{G}$, the present result follows immediately from Theorem 6.5.

It may be noted that $(Y, T(\mathcal{G}))$ is always an LTS.

Since both $R$ and $C$ are normed linear spaces and $R$ is a locally convex real LTS, the preceding theorems may be applied to the adjoint space $C(X, R)$ or $C(X, C)$ of an LTS $X$, for which we use the usual notation $X^{*}$. Thus we see that $P^{w} X_{a}^{*}$ is the adjoint of the product $P X_{a}$ in its product topology, and that there are several "interesting" topologies for $P^{w} X_{a}$ under which its adjoint is $P X_{a}^{*}$.

When each $Y_{a}$ is the same space $Y$, we write $Y^{A}$ for $P Y_{a}$ and $Y_{w}^{A}$ for $P^{w} Y_{a}$. With this notation, our principal theorem becomes:

9.5. TheOREM. Let $A$ be $a$ set and $Y$ an LTS. Let $u$ be the product topology for $Y^{A}$ and $J$ a topology for $Y_{w}^{A}$ such that $T(W) \subset J \subset T(\mathfrak{T})$, where, in the definition of $T(W)$, we take $X=\left(Y^{*}\right)^{A}$. Then $\left(Y^{*}\right)_{w}^{A}$ is isomorphic to $\left(Y^{A}, \mathcal{u}\right)^{*}$ and $\left(Y^{*}\right)^{A}$ is isomorphic to $\left(Y_{w}^{A}, \mathfrak{J}\right)^{*}$. In either case, an isomorphism $\mu$ is defined by $[\mu(f)](y)=\sum\left\{f_{a}\left(y_{a}\right): a \in A\right\}$.

The general form of the duality theorem for products of linear spaces is as follows, with the obvious specializations to adjoints and products of a space with itself.

9.6. THEOREM. Let $Z$ be an LTS without arbitrarily small linear subspaces. For each $a$ in a set $A$, let $X_{a}$ and $Y_{a}$ be $Z$-dual LTS, with a connecting bihomomorphism $\Omega^{a}$. Further, let $u$ be the product topology for $P X_{a}$ and $\Im$ a topology for $P^{w} Y_{a}$ such that $T(\mathfrak{W}) \subset J C T(\mathfrak{T})$. Then $\left(P X_{a}, \mathcal{U}\right)$ and $\left(P^{w} Y_{a}, \mathfrak{J}\right)$ are $Z$-dual, under the bihomomorphism $\Omega$ defined as in (2).

REMARK. If we take $Z=R$ or $C$ and $J=T(\mathcal{G})$ in this theorem, we obtain the main theorem of Katêtov's paper [5, Theorem 2.2]. We note that in his 
hypothesis that all the spaces are locally-convex and Hausdorff is not essential to the theorem.

10. Self-adjoint spaces. Of special interest is the case in which $Y_{a}^{*}=Y_{a}$ for each $a \in A$. In this category, perhaps the most important are the Hilbert spaces. If $H$ is a real Hilbert space, the usual inner product of $H$ is a bihomomorphism under which $H$ is an $R$-complement of itself, as is well-known. For this type of space, we have the following special form of the duality theorem.

10.1. TheOREM. For each a in some set $A$, let $H_{a}$ be a real Hilbert space with inner product $(p, q)_{a}$. If $P H_{a}$ is given the product topology and $P^{w} H_{a}$ has a topology $\mathfrak{J}$ such that $T(\mathfrak{W}) \subset J \subset T(\mathfrak{T})$, these two spaces are adjoints of each other, connected by the bihomomorphism $\Omega(x, y)=\sum\left\{\left(x_{a}, y_{a}\right)_{a}: a \in A\right\}$.

Since a real Hilbert space is also its own adjoint in the weak topology, either this or the norm topology may be used in any $H_{a}$ in taking the products above. A result analogous to 10.1 is true for complex Hilbert spaces, requiring only a slight modification of our earlier arguments; the proof will be omitted. $4]$.

From 10.1 we readily obtain the following result of Dixmier [2, Theorem

10.2. Corollary. Let $H$ be a Hilbert space with inner product $(x, y)$; let $B$ be the family of all bounded linear operators on $X$. Then for any linear functional $L$ on $B$, these are equivalent:

(1) $L$ is continuous in the weak topology;

(2) $L$ is continuous in the strong topology;

(3) There are elements $x_{1}, x_{2}, \cdots, x_{n}$ and $y_{1}, y_{2}, \cdots, y_{n}$ in $H$ such that $L(f)=\sum_{i=1}^{n}\left(f\left(x_{i}\right), y_{i}\right)$, for all $f \in B$.

Proof. Clearly (1) implies (2) and (3) implies (1). To show that either (1) or (2) implies (3), we note that $H^{H}$, in either the weak or the strong topology is a locally-convex Hausdorff LTS; therefore, by a well-known result (see Wehausen [7, Theorem 11]), any continuous linear functional $L$ on $B$ can be extended to a continuous linear functional $L^{\prime}$ on $H^{H}$. By Theorem 10.1, $L^{\prime}$ may be considered to be a member of $H_{w}^{H}$; specifically, there are points $x_{1}, x_{2}, \cdots, x_{n}$ in $H$ and a function $G$ on $H$ to $H$ such that $L^{\prime}(f)=\sum_{i=1}^{n}\left(f\left(x_{i}\right)\right.$, $\left.G\left(x_{i}\right)\right)$ for all $f \in H^{H}$. Putting $y_{i}=G\left(x_{i}\right)$ for each $i$ gives (3).

The spaces $R$ and $C$ are the one-dimensional Hilbert spaces, with inner product $(x, y)=x y$ or $x \bar{y}$, respectively. Consequently, the following theorem holds.

10.3. ThEOREM. Let $A$ be any set. If $R^{A}\left[C^{A}\right]$ has the product topology and $R_{w}^{A}\left[C_{w}^{A}\right]$ has the topology $T(w)$ or $T(\mathcal{G})$, then these two spaces are adjoints of each other, connected by the bihomomorphism $\Omega$ defined by $\Omega(x, y)=\sum\left\{x_{a} y_{a}\right.$ : $a \in A\}$ or $\left.\sum x_{a} \bar{y}_{a}: a \in A\right\}$, respectively. 
For the case in which $A=I$, the set of positive integers, this includes Banach's classical theorem $[1$, p. 50$]$ on the general linear functional on the space $(s)$ of all real sequences.

\section{REFERENCES}

1. S. Banach, Théorie des opérations linéaires, Subwensji Funduszu kultury narodowey, Warsaw, 1932, p. 50.

2. J. Dixmier, Les fonctionnelles linéaires sur l'ensemble des opérateurs bornés d'un espace de Hilbert, Ann. of Math. vol. 51 (1950) pp. 387-408.

3. D. H. Hyers, Locally bounded linear topological spaces, Revista de Ciencias vol. 41 (1939) pp. 558-574.

4. S. Kaplan, Extensions of the Pontrjagin duality, I: Infinite products, Duke Math. J. vol. 15 (1948) pp. 649-658.

5. M. Katětov, On convex topological linear spaces, Acta Facultatis Rerum Naturalium Universitatis Carolinae, Prague, no. 181 (1948) pp. 1-20.

6. G. Köthe, Die Quotientenräume eines linear vollkommen Raumes, Math. Zeit. vol. 51 (1947) pp. 17-35.

7. J. V. Wehausen, Transformations in linear topological spaces, Duke Math. J. vol. 4 (1938) pp. 157-169.

The Tulane University of Louisiana, New Orleans, La.

Florida State University, Tallahassee, Fla. 